\title{
Modeling Fairness in Resource Allocation for Secondary Users in a Competitive Cognitive Radio Network
}

\author{
Lutfa Akter, Student Member, IEEE and Balasubramaniam Natarajan, Senior Member, IEEE \\ WiCom Group, Department of Electrical \& Computer Engineering \\ Kansas State University, Manhattan, Kansas 66506-5204, \\ Telephone: (785) 532-4664, Email: lutfa@ksu.edu, bala@ksu.edu
}

\begin{abstract}
We consider a multi-channel cognitive radio network (CRN) where multiple secondary users share a single channel and multiple channels are simultaneously used by a single secondary user (SU) to satisfy their rate requirements. In this competitive CRN, our interest is in determining optimal power and rate distribution choices for each $S U$ while maintaining fairness in "quality of experience" across all SUs. Unlike prior approaches that focus on resource allocation based on instantaneous quality of service (QoS), our approach to fairness encompasses both current and prior history of user experience with respect to QoS. Specifically, we quantify user experience over time by introducing dynamic fairness weights for each $\mathrm{SU}$ in the resource allocation framework. The dynamics of the weights are governed by the Homo Egualis (HE) society model. We consider Jain system level fairness index [1] as a measure of fairness in resource allocation. Simulation results show that the weighted resource allocation scheme provide a better system level fairness index relative to the unweighted allocation scheme.
\end{abstract}

\section{INTRODUCTION}

Any secondary user in a cognitive radio network (a network of secondary users) continuously senses the absence/presence of primary users in its intended spectrum and may use the spectrum when primary users are absent. All SUs maintain QoS through the transmission duration by dynamically seeking out the best transmission parameters (e.g., channel, rate, transmit power). In a competitive CRN where multiple SUs compete with each other for resources (transmit power, rate, channel), fairness in resource allocation among SUs plays an important role. Prior research efforts have focused on a wide range of issues in CRN including sensing of primary users [2]-[3], finding optimal channel, transmit power, modulation type and rate to SUs [4]-[5], fairness among SUs in opportunistic spectrum access/scheduling [6]-[9] and fairness in transmit power allocation to SUs [10]. In this work, our objective is to allocate resources for SUs in a competitive CRN maintaining fairness based on both instantaneous and past user experiences with respect to QoS (collectively referred to as "quality of experience"). In the following paragraphs, we provide an overview of prior work in fair resource allocation and scheduling, and highlight the contribution of our work.

A fair random access (in terms of airtime share) protocol for dissimilar radio systems in open spectrum access scenario is studied in [6]. In their proposed fair random access protocol,

978-1-4244-6557-6/10/\$26.00 (C) 2010 IEEE each radio system contends for the spectrum with a finite probability. The authors also propose a HE society model based distributed approach to determine the contending probability. A fair opportunistic spectrum access based on fast catchup strategy that reduces the first passage time (first passage time is the amount of time after which all SUs have equal access right to the available channels) is studied in [7]. In [8], the authors study three variants of utility functions Maxsum-Reward, Max-min-Reward and Max-Proportional Fair to allocate spectrum in CRN under protocol interference model. In [9], the authors study the three joint spectrum allocation and scheduling methods such as MAximum throughput Spectrum allocation and Scheduling (MASS), Max-min fair MAximum throughput Spectrum allocation and Scheduling (MMASS) and Proportional fAir Spectrum allocation and Scheduling (PASS) in CRN with the objective to achieve a tradeoff between throughput and fairness while ensuring interference-free transmission at any time (taking into account both protocol and physical interference models). While significant efforts have gone into fair spectrum allocation, the authors in [6]-[9] have not considered fairness in spectrum access and dynamic power/rate management in an integrated framework.

In [10], the authors find optimal transmit power for users in wireless cellular and ad hoc networks considering proportional and minmax fairness. In proportional fairness optimization problem formulation, the authors consider a static weight for each user and use the weight into optimization problem. In minmax fairness optimization problem formulation, the transmit power that maximizes the minimum signal to interference ratio is determined. However, the authors in [10] do not explicitly evaluate user level fairness or system level fairness for their proposed resource allocation schemes.

Though there have been significant research efforts in the areas of resource allocation and fairness among SUs in open spectrum access/scheduling, resource allocation while maintaining fairness in "quality of experience" among SUs has not been investigated. We consider a system model where (1) multiple channels each with different quality is available for opportunistic use by multiple SUs; (2) more than one SU may coexist in every channel and each SU needs to use multiple channels to satisfy their rate requirements, and (3) measure for QoS include BER and minimum rate requirement. In such an environment, we determine transmit power and rate that each SU needs to employ in its intended channels while 
maintaining fairness across SUs. Unlike prior efforts that focus on fair scheduling of SUs and interference management for primary users, we consider an alternate framework. We assume scheduling is complete and SUs have identified the channels to use. Since we allow more than one SU to coexist in a channel, the QoS experience of SUs will be different from each other and may vary with time. To achieve fairness in "quality of experience" across SUs, we associate dynamic fairness weights for each SU in the resource allocation scheme. The dynamic fairness weights capture the user's present and past experiences with respect to QoS and evolve based on the Homo Egualis (HE) society attitude. HE based fairness weights result in a proportionate resource allocation with respect to users demand. Hence, the fairness weights in the resource allocation scheme cause some users to sacrifice resources and some users to gain resources to maintain a balance in allocated resources. We consider Jain system level fairness index [1] to quantify fairness in resource allocation. Simulation results show that the inclusion of dynamic fairness weight in the resource allocation scheme provides a better system level fairness index relative to the unweighted resource allocation scheme.

\section{SYSTEM MODEL}

We consider a CRN with a total of $M$ secondary users and $L$ free channels available for opportunistic use (determined after spectrum sensing) by multiple SUs. We assume that each channel can be used simultaneously by multiple secondary users via some form of non-orthogonal multiple access scheme, and a single secondary user can use several channels at the same time to meet their rate requirements. Our interest in this work is to maintain QoS and fairness for these competing SUs via effective resource allocation. We consider BER and minimum rate requirement as measures to indicate QoS. In order to enable mathematical tractability of the optimization framework, we invoke the following assumptions: (1) we assume that we have a central cognitive network controller that will perform the resource allocation and has access to all SUs channel and interference parameters; (2) every active SU radio has an upper limit on power and rate (bits/channel use) at which it can transmit; (3) all SUs employ M-ary QAM modulation scheme with an adaptable modulation order $\mathbf{M}$; (4) simple path loss model for channel has been assumed; (5) each channel has a maximum rate (bits/channel use) that it can support, and (6) each user has a minimum rate and BER that needs to be maintained. Additionally, we enforce an interference temperature threshold to protect possible primary user transmission on any channel.

Under this system model, we determine transmit power and rate while maintaining fairness on present and past quality of user experiences with respect to QoS. We introduce dynamic fairness weights for each user into resource allocation scheme and develop evolution models for the fairness weights that captures the present and past quality of user experiences. Table I defines all relevant terms (at $n$-th time instant) used throughout the paper.
TABLE I

NOTATIONS

\begin{tabular}{c|c}
\hline \hline$\sigma^{2}(n, k)$ & Noise variance in $k$-th channel \\
\hline$\rho_{j, i}(n)$ & Orthogonality factor between users $j$ and $i$ \\
\hline$h_{i, i}(n, k)$ & Power gain from $i$-th transmitter to $i$-th receiver in $k$-th channel \\
\hline$h_{i, m}(n, k)$ & Power gain from $i$-th transmitter at location $m$ in $k$-th channel \\
\hline$p_{i}(n, k)$ & Transmit power per bit of $i$-th user in $k$-th channel \\
\hline$p_{i}^{\text {max }}(n, k)$ & Maximum transmit power per bit of $i$-th user in $k$-th channel \\
\hline$I_{t}^{u}(n, k)$ & Interference temperature constraint in $k$-th channel \\
\hline$b_{i}(n, k)$ & Rate of $i$-th user in $k$-th channel \\
\hline$b_{i}^{\text {max }}(n, k)$ & Maximum rate of $i$-th user in $k$-th channel \\
\hline$R_{c h}^{u}(n, k)$ & Maximum rate supported by $k$-th channel \\
\hline$R_{i}^{l}(n)$ & Minimum required rate for $i$-th user \\
\hline$p_{e, i}(n, k)$ & BER for $i$-th user in $k$-th channel \\
\hline$p_{e, i}^{u}(n)$ & BER threshold at receiver for $i$-th user in any channel \\
\hline$\gamma_{i}(n, k)$ & SINR per bit for $i$-th user in $k$-th channel \\
\hline \hline
\end{tabular}

In the following section, we describe the resource allocation framework that is considered in this work.

\section{Resource Allocation Framework}

The objectives of the resource allocation framework are to (1) minimize the total transmit power, and (2) maximize the total rate while satisfying the QoS requirements and maintaining fairness across all active SUs. The mathematical description of the bi-objective optimization corresponds to:

$$
\begin{aligned}
\text { Determine } & {\left[\mathbf{p}^{T}(n) \mathbf{b}^{T}(n)\right]^{T} } \\
\text { where, } & \mathbf{p}(n)=\left[p_{1}(n, 1), \cdots, p_{M}(n, L)\right]^{T} \text { and } \\
& \mathbf{b}(n)=\left[b_{1}(n, 1), \cdots, b_{M}(n, L)\right]^{T} \\
\text { To Minimize: } & F_{1}=\sum_{k=1}^{L} \sum_{i=1}^{M} w_{i}^{p}(n) p_{i}(n, k) \text { and } \\
& \\
\text { Maximize: } & F_{2}=\sum_{k=1}^{L} \sum_{i=1}^{M} w_{i}^{b}(n) b_{i}(n, k)
\end{aligned}
$$

subject to

$$
\begin{array}{ll}
C 1: & 0 \leq p_{i}(n, k) \leq p_{i}^{\max }(n, k), \forall i, k \\
C 2: & b_{i}(n, k) \in\left[1, \cdots, b_{i}^{\max }(n, k)\right], \forall i, k \\
C 3: & \sum_{i=1}^{M} p_{i}(n, k) h_{i, m}(n, k) \leq I_{t}^{u}(n, k), \forall k \\
C 4: & \sum_{i=1}^{M} b_{i}(n, k) \leq R_{c h}^{u}(n, k), \forall k \\
C 5: & \sum_{k=1}^{L} b_{i}(n, k) \geq R_{i}^{l}(n) \\
C 6: & p_{e, i}(n, k) \leq p_{e, i}^{u}(n), \forall i, k .
\end{array}
$$

where

$$
\begin{array}{r}
p_{e, i}(n, k) \leq \frac{4}{b_{i}(n, k)} Q\left(\sqrt{\frac{3 b_{i}(n, k) \gamma_{i}(n, k)}{\left(2^{b_{i}(n, k)}-1\right)}}\right), \\
\quad \text { for odd } b_{i}(n, k) ;
\end{array}
$$




$$
\begin{gathered}
p_{e, i}(n, k)=\frac{4}{b_{i}(n, k)}\left(1-2^{-\frac{b_{i}(n, k)}{2}}\right) \\
\quad \times Q\left(\sqrt{\frac{3 b_{i}(n, k) \gamma_{i}(n, k)}{\left(2^{b_{i}(n, k)}-1\right)}}\right) \\
\text { for even } b_{i}(n, k) ; \\
\gamma_{i}(n, k)=\frac{p_{i}(n, k) h_{i, i}(n, k)}{\sum_{j=1, j \neq i}^{M} p_{j}(n, k) h_{j, i}(n, k) \rho_{j, i}^{2}(n)+\sigma^{2}(n, k)} .
\end{gathered}
$$

Here, $w_{i}^{p}(n)$ and $w_{i}^{b}(n)$ are the dynamic fairness weights for user $i$ based on allocation of transmit power and rate till time instant $(n-1)$, respectively; constraints $C 1$ and $C 2$ indicate limits on transmit power and rate, respectively; $C 3$ indicates the interference temperature constraint; $C 4$ indicates the total rate supported by a channel; $C 5$ represents the required rate of users and finally $C 6$ is QoS/BER constraint. Since $b_{i}(n, k)$ is discrete and constraint $C 6$ is nonlinear, the optimization formulation presented above is a constrained multi-objective mixed integer nonlinear programming (multiobjective MINLP) resource allocation scheme, which is NPhard in general. Relaxing the integer constraint on rate, $b_{i}(n, k)$ (as assumed in [11]) and assuming $b_{i}(n, k)$ as continuous variable, the above resource allocation scheme can be restated with $C 2$ as:

$$
C 2: 1 \leq b_{i}(k) \leq b_{i}^{\max }(k), \forall i, k .
$$

As in [5], constraint $C 6$ can be written as

$$
C 7: \quad-\gamma_{i}(n, k) \leq-C_{q a r g}(n)\left(2^{b_{i}(n, k)}-1\right), \forall i, k ;
$$

where, $C_{\text {qarg }}(n)$ is a constant and is also determined following the analysis in [5]. The resource allocation scheme with combined single objective can be rewritten as:

$$
\begin{array}{r}
\text { Minimize } \tau_{1} F_{1}-\tau_{2} F_{2} \\
\text { subject to } \\
C 1, C 2, C 3, C 4, C 5, C 7 .
\end{array}
$$

The parameters $\tau_{1}$ and $\tau_{2}$ in the combined objective function are the scalarization factors and can be set following the discussion in [12]. Finally, we use the solution obtained from the convex formulation (Eq. (7)) as a starting point to search in the neighborhood for the optimal discrete valued $b_{i}(n, k)$ (denoted as $\mathbf{b}^{\mathbf{o p t}}$ ). Based on the new discrete solution, the optimal transmit power $\mathbf{p}^{\mathbf{o p t}}$ is recalculated using Eq. (6).

In the following section, we describe the analogy between the social behavior of human beings and that of SUs in CRN. We then use the society model to design the evolution models for the fairness weights $w_{i}^{p}(n)$ and $w_{i}^{b}(n)$.

\section{Homo Egualis (HE) Society Model}

The secondary users in a CRN may behave rationally while competing and cooperating for resources, survival and social efficiency just like human beings in society [13]. Hence, secondary users behavior in CRN can be modeled based on human society model.
In many decision-making and strategy-settings people do not behave like the self-interested "rational" actor depicted in neoclassical economics and game theory [14]. In a Homo Egualis society, individuals have an inequality aversion. As a result altruists appear in ultimatum and public games. As Gintis states in [14], support for Homo Egualis comes from the anthropological literature describing how Homo Sapiens evolved in small hunter-gatherer groups. Such societies had no centralized structure of governance, so the enforcement of norms depends on the voluntary participation of peers. A Homo Egualis Society can be modeled following [14] where the utility function of player $m, u_{m}$ in an $M$-player game is:

$u_{m}=z_{m}-\frac{\alpha_{m}}{M-1} \sum_{o=1, z_{o}>z_{m}}^{M}\left(z_{o}-z_{m}\right)-\frac{\beta_{m}}{M-1} \sum_{z_{o}<z_{m}}\left(z_{m}-z_{o}\right)$

where $\mathbf{z}=\left[z_{1}, \cdots, z_{m}, \cdots, z_{M}\right]^{T}$ is the pay-off vector of the players and $0 \leq \beta_{m}<\alpha_{m}<1$. In Eq. (8), considering $\beta_{m}$ less than $\alpha_{m}$ in the utility model reflects the fact that Homo Egualis exhibits a weak urge to inequality when doing better than the others and a strong urge to reduce inequality when doing worse that the others. In [14], it is also shown that in this model the salient behaviors in ultimatum and public games, where fairness does matter, can be reproduced.

In the following section, we develop an evolution model for $w_{i}^{p}(n)$ and $w_{i}^{b}(n)$ following the concept of HE society model.

\section{UNDERSTANDING AND MODELING OF FAIRnESS}

A resource allocation framework (as an example, resource allocation framework in [5]) for the system model described in Section II provides the optimal transmit power and rate across the channels for all SUs for a given time instant. If the underlying optimization problem is convex, resource allocation is optimal. However, users may not be satisfied with optimal allocation of resources based on instantaneous QoS. An example of dissatisfaction among SUs may arise when two SUs with different minimum rate requirements are allocated the same rate. Another example of dissatisfaction among SUs may arise when a user is assigned higher average power per bit relative to other users. Typically dissatisfaction is a feeling that develops over time. Hence, fairness in terms of current and prior history of user satisfaction with respect to QoS in optimal resource allocation is an important consideration. We define two fairness metrics, one based on instantaneous average power per bit $p_{i}^{o p t}(n, k)$ and another based on instantaneous allocated rate $b_{i}^{o p t}(n, k)$ for user $i$ as

$$
x_{i}^{p}(n)=\frac{1}{L} \sum_{k=1}^{L} p_{i}^{o p t}(n, k)
$$

and

$$
x_{i}^{b}(n)=\frac{R_{i}^{l}(n)}{\sum_{k=1}^{L} b_{i}^{o p t}(n, k)} .
$$

Equation (9) tells that a lower value of $x_{i}^{p}(n)$ means a favorable power allocation from the resource allocation scheme to 
a user. Equation (10) tells that $x_{i}^{b}(n)$ can take value between 0 to 1 . It is to be noted that $x_{i}^{b}(n)$ with value close to 0 indicates a comparatively higher allocated rate to minimum requirement (favorable rate allocation to a user) and $x_{i}^{b}(n)$ with value close to 1 indicates a comparatively lower allocated rate to minimum requirement. The fairness weights $w_{i}^{p}(n)$ and $w_{i}^{b}(n)$ are modeled as a function of metrics $x_{i}^{p}(n)$ and $x_{i}^{b}(n)$, respectively, following the concepts in HE society model. The metrics $x_{i}^{p}(n)$ and $x_{i}^{b}(n)$ into fairness weights $w_{i}^{p}(n)$ and $w_{i}^{b}(n)$, respectively, capture the current experience and the evolution models of $w_{i}^{p}(n)$ and $w_{i}^{b}(n)$ capture the past experience with respect to QoS.

The system level fairness as in [1] at time instant $n$ can be defined as

$$
\text { fairness index }(n)=\frac{1}{M} \frac{\sum_{i=1}^{M} x_{i}(n)}{\sum_{i=1}^{M} x_{i}^{2}(n)} \sum_{i=1}^{M} x_{i}(n),
$$

where, $x_{i}(n)=x_{i}^{p}(n)$ or $x_{i}^{b}(n)$. It is also to be noted that system fairness index can take value from 0 to 1 . An index 0/1 means that system is totally unfair/fair in allocation. An index close to 1 results when $x_{i}(n)$ of all users are comparable and close to 1 . As an example, for a system with three users $(M=$ $3)$, an allocation scheme that results $x_{1}(n), x_{2}(n)$ and $x_{3}(n)$ as $0.85,0.75$ and 0.60 , respectively (system fairness index value is computed as 0.98) is more fair than the allocation scheme that results $x_{1}(n), x_{2}(n)$ and $x_{3}(n)$ as $1.00,0.90$ and 0.30 , respectively (system fairness index value is computed as 0.85 ). This is because $0.85,0.75$ and 0.60 has lower variance than $1.00,0.90$ and 0.30 .

The evolution model for the fairness weights $w_{i}^{p}(n)$ and $w_{i}^{b}(n)$ based on HE society model are shown in Algorithm 1. In Algorithm 1, $n^{\max }$ represents considered time horizon. In the first time instant, fairness weight vectors, $\mathbf{w}^{p}(n)$ and $\mathbf{w}^{b}(n)$ are initialized to $\mathbf{1}$. In the following time instants, based on relative values of current quality of experiences $x_{i}^{p}(n)$ and $x_{i}^{b}(n)$, weights $w_{i}^{p}(n)$ and $w_{i}^{b}(n)$ are updated. A relatively higher value of $x_{i}^{p}(n)$ and $x_{i}^{b}(n)$ (with respect to other users) result $w_{i}^{p}(n)$ and $w_{i}^{b}(n)$ to be a higher value. A higher value of $w_{i}^{p}(n)$ and $w_{i}^{b}(n)$ causes more importance on minimizing power and maximizing allocated rate, respectively, in next time instant. For a user $i$, a smaller value for $\beta_{m}$ than $\alpha_{m}$ indicates a weak urge to reduce $w_{i}^{p}(n)$ and $w_{i}^{b}(n)$ (as it reduces importance on minimizing power and maximizing allocated rate, respectively, in next time instant). The criteria $\beta_{m}<\alpha_{m}$ captures the Homo Egualis society attitude among the SUs in CRN.
TABLE II

Channel Quality Parameters

\begin{tabular}{c|c|c|c|c|c|c|c|c}
\hline \hline Channel, $k$ & 1 & 2 & 3 & 4 & 5 & 6 & 7 & 8 \\
\hline$\sigma^{2}(n, k),\left(\times 10^{-3}\right)$ & 5 & 4 & 3 & 2 & 2.5 & 6 & 4 & 4 \\
\hline \hline
\end{tabular}

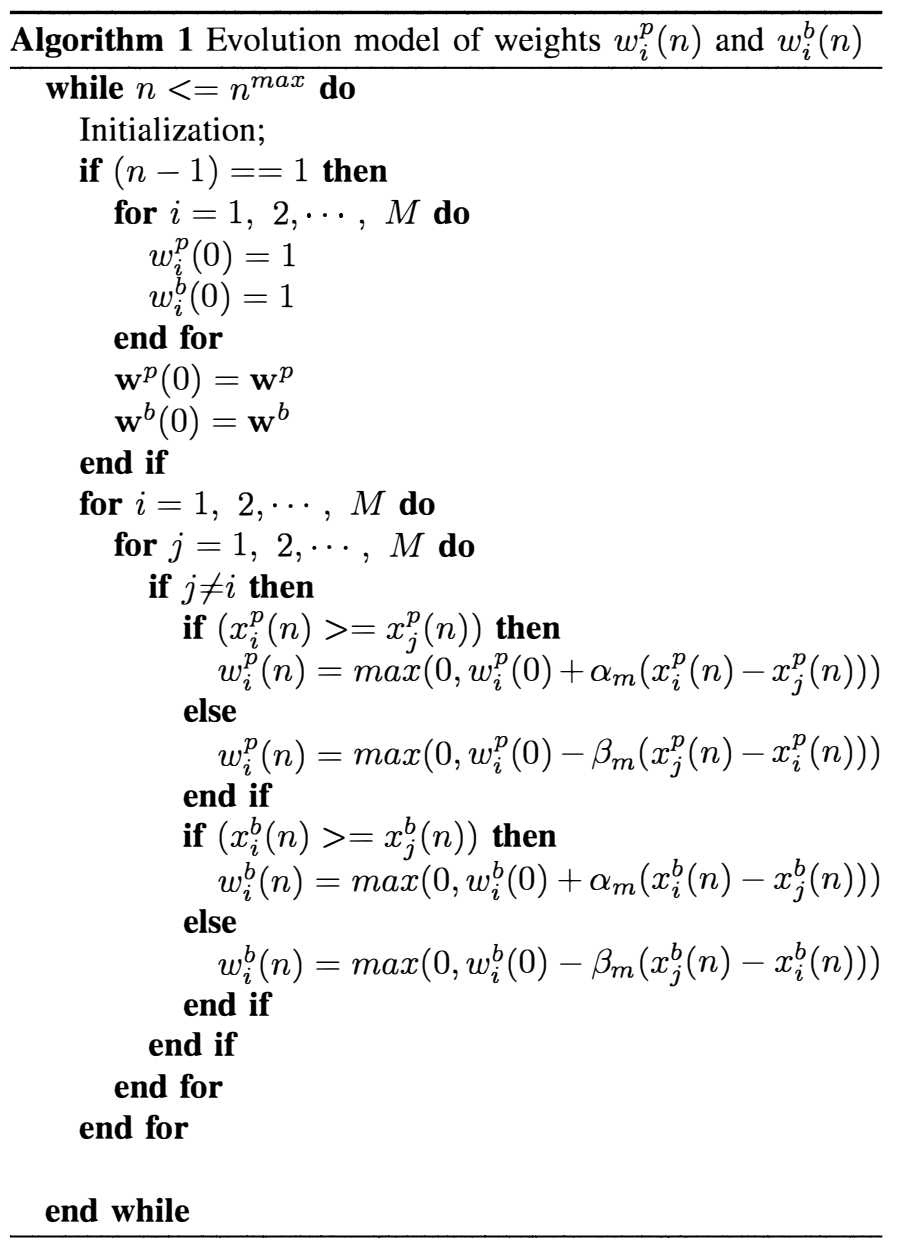

\section{Numerical RESUlts}

In this section, we evaluate the impact of introducing dynamic fairness weight in the resource allocation framework. We assume a CRN with $L=8$ available channels and a total of $M=9$ secondary users. Table II provides information on the channel quality for all $L$ channels. Table III lists the minimum rate requirement for each SU. Finally, Table IV contains all other system parameters that are relevant to our resource allocation framework. Based on all this information, our objective is to find the optimal transmit power and rate that each of the $M$ SUs should employ to achieve fairness in "quality of experience."

The resource allocation scheme has one non-linear constraint (constraint $C 7$ ); we use "Sequential Quadratic Programming (SQP)" method to solve the problem. It is to be noted that unweighted resource allocation scheme corresponds to the case of assuming $w_{i}^{p}(n)=1, \forall i, n$ and $w_{i}^{b}(n)=1, \forall i, n$ in the resource allocation scheme. Figures 1(c), 1(d), and 2(c), 
TABLE III

Minimum RATE REQUiREMENT OF USERS

\begin{tabular}{c|c|c|c|c|c|c|c|c|c}
\hline \hline User, $i$ & 1 & 2 & 3 & 4 & 5 & 6 & 7 & 8 & 9 \\
\hline$R_{i}^{l}(n)$ & 12 & 8 & 10 & 9 & 15 & 16 & 13 & 11 & 10 \\
\hline \hline
\end{tabular}

TABLE IV

SySTEM PARAMETERS

\begin{tabular}{c|c}
\hline \hline$p_{i}^{\text {max }}(n, k) \forall i, k$ & 5 \\
\hline$b_{i}^{\text {max }}(n, k) \forall i, k$ & 6 \\
\hline$p_{e, i}^{u}(n) \forall i$ & $10^{-3}$ \\
\hline$I_{t}^{u}(n, k) \forall k$ & $200 \times \sigma^{2}(n, k)$ \\
\hline$R_{c h}^{u}(n, k) \forall k$ & 30 \\
\hline$\rho_{j, i}(n)$ & 0.03125 \\
\hline$n^{\text {max }}$ & 50 \\
\hline$\alpha_{m}$ & 0.35 \\
\hline$\beta_{m}$ & 0.15 \\
\hline \hline
\end{tabular}

2(d) present the short term averaged power and rate allocated for users 2 and 5 from weighted and unweighted resource allocation schemes, respectively with time $\left(\tau_{1}=\tau_{2}=0.5\right)$. Evolution of weights $w_{2}^{p}(n), w_{2}^{b}(n)$ and $w_{5}^{p}(n), w_{5}^{b}(n)$ are also shown in Figs. 1(a), 1(b) and 2(a), 2(b), respectively.

Figures 1(d) and 2(d) show that a decreasing fairness weight (with respect to 1) with time (as shown in 1(b)) results in smaller allocated rate; whereas an increasing fairness weight (with respect to 1) with time (as shown in 2(b)) results in an higher allocated rate. Figures 1(d) and 2(d) also show that allocated rate with dynamic fairness weight is smaller for user 2 and higher for user 5 compared to that obtained from unweighted resource allocation. This indicates that proposed fairness weight in the resource allocation scheme results in sacrificing rate for user 2 and gaining more rate for user 5 to achieve a rational resource allocation. That is, dynamic fairness weights $w_{i}^{p}(n)$ and $w_{i}^{b}(n)$ promote cooperative, rational attitude of SUs in CRN like human beings in Homo Egualis Society. Similar impact of the weights are observed on short term averaged rate allocated to other users in the CRN.

Figures 1(c) and 2(c) depict that allocated power is insensitive to fairness weight (shown in Figs. 1(a) and 2(a)) for both users. This can be explained as follows. In order to satisfy constraint $C 7$, one can increase power, $p_{i}(n, k)$ or decrease rate, $b_{i}(n, k)$. Equation (6) suggests that varying rate, $b_{i}(n, k)$ is more effective. This is because $\gamma_{i}(n, k)$ is linearly related to $p_{i}(n, k)$ while rate $b_{i}(n, k)$ is an exponent of denominator in constraint $C 7$. Therefore, for a given BER constraint, optimization engine prefers to vary $b_{i}(n, k)$ instead of $p_{i}(n, k)$ to satisfy the constraint. Hence, changing $w_{i}^{b}(n)$ has stronger impact than $w_{i}^{p}(n)$ in the resource allocation scheme.

Figure 3(a) shows the long term averaged transmit power allocated across users $\left(\tau_{1}=\tau_{2}=0.5\right)$. From Fig. 3(a), we observe that transmit power allocated from weighted resource allocation scheme is same (as expected due to the reason mentioned in short term averaged power variation) compared to unweighted allocation scheme. Figure 3(b) illustrates the long term averaged rate allocated across users $\left(\tau_{1}=\tau_{2}=0.5\right)$. From Fig. 3(b), we see almost equal rate is allocated from unweighted scheme across users irrespective of their demand. Whereas the fairness weights in the resource allocation scheme cause some users $\{1,5,6,7\}$ to sacrifice resources and some users $\{2,3,4,8,9\}$ to gain resources to maintain a balance in allocated resources and their demand as observed in short term averaged allocated rate. Therefore, long term averaged rate allocated across users also reflect the cooperative, rational attitude of SUs in CRN as expected, and observed in short term averaged rate from introducing HE society based dynamic fairness weights in allocation scheme.

Figure 4 presents the short term averaged system level fairness index for power. We see from Fig. 4 that system level fairness index of both weighted and unweighted allocation schemes are same which is expected as allocated power is insensitive to inclusion of fairness weight in the resource allocation scheme. Figure 5 shows the long term averaged system level fairness index for rate. From Fig. 5, we see that system level fairness index of the weighted allocation scheme is higher than the unweighted scheme. This can be explained as follows. The weighted allocation scheme promotes equality across users unlike greedy attitude in unweighted scheme. That is, $x_{i}^{b}(n)$ takes value close 1 in the weighted scheme as designed in Eq. (10). Therefore, fairness index of weighted allocation scheme becomes higher i.e., more closer to 1 compared to unweighted scheme.
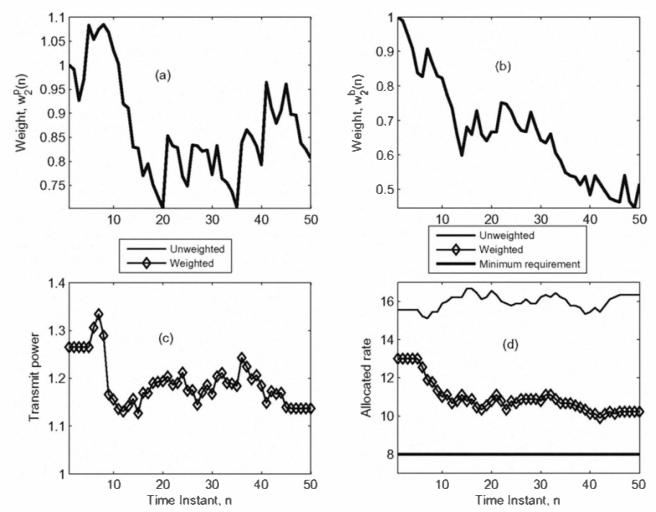

Fig. 1. Short term averaged transmit power and rate allocated to user 2 from weighted and unweighted resource allocation schemes, respectively.

\section{CONCLUSION}

In this paper, we determine optimal transmit power and rate distribution that each SU needs to employ in a multi-channel CRN considering current and past history of user experience with respect to QoS. We introduce fairness weights for each user that captures current and past history of user experience and design evolution models for the fairness weights based on HE society model. We consider Jain system level fairness 

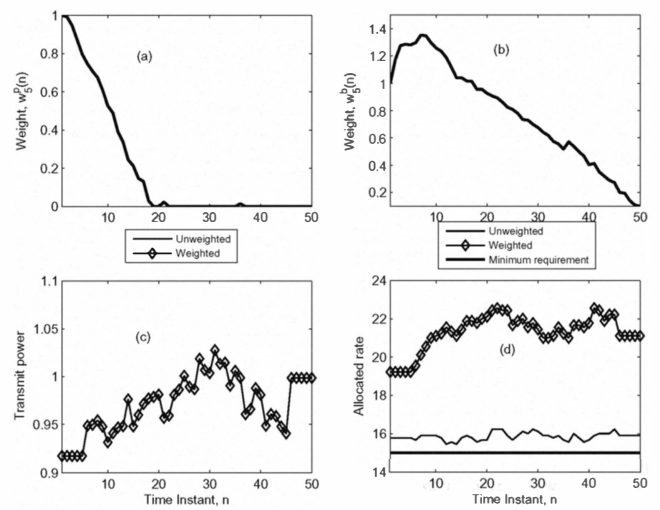

Fig. 2. Short term averaged transmit power and rate allocated to user 5 from weighted and unweighted resource allocation schemes, respectively.
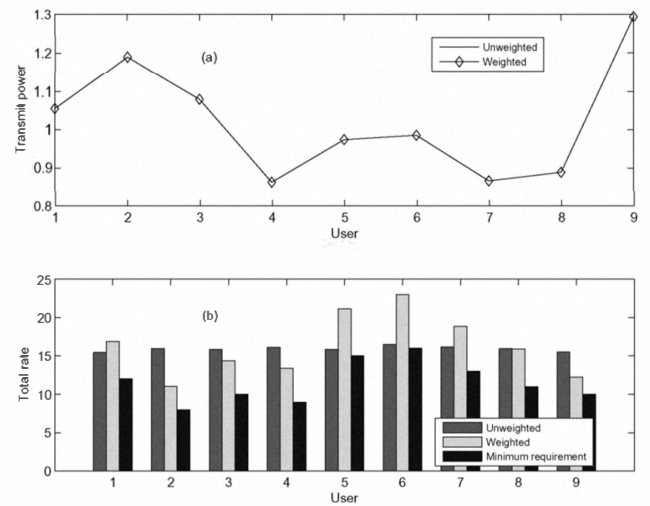

Fig. 3. Long term averaged transmit power and rate allocated across users from weighted and unweighted resource allocation schemes, respectively.

index as a measure of fairness in resource allocation scheme. Simulation results illustrate that incorporating dynamic fairness weights in the resource allocation scheme provide better system level fairness index compared to the unweighted resource allocation scheme.

\section{REFERENCES}

[1] R. K. Jain, D. W. Chiu and W. R. Hawe, "A Quantitive Measure of Fairness and Discrimination for Resource Allocation in Shared Computer Systems," 1984.

[2] D. Cabric, S. M. Mishra and R. W. Brodersen, "Implementation Issues in Spectrum Sensing for Cognitive Radios," IEEE Asilomar Conference on Signals, Systems and Computers, Vol. 1, pp. 772-776, November, 2004.

[3] A. Ghasemi and E. S. Sousa, "Optimization of Spectrum Sensing for Opportunistic Spectrum Access in Cognitive Radio Network," IEEE Consumer Communications and Networking Conference, pp. 1022 1026, January, 2007.

[4] T. R. Newman, B. A. Barker, A. M. Wyglinski, A. Agah, J. B. Evans and G. J. Minden "Cognitive Engine Implementation for Wireless Multicarrier Transceivers," Wiley Journal on Wireless Communications and Mobile Computing, vol. 7(9), November, 2007.

[5] L. Akter and B. Natarajan, "QoS Constrained Resource Allocation to Secondary Users in Cognitive Radio Networks," Computer Communications, June, 2009, Elsevier.

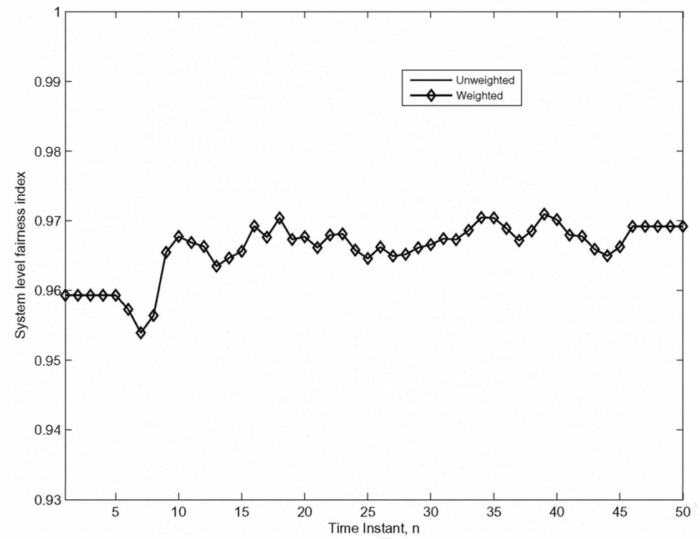

Fig. 4. Comparison of short term averaged system level fairness index (based on power) of weighted and unweighted resource allocation schemes.

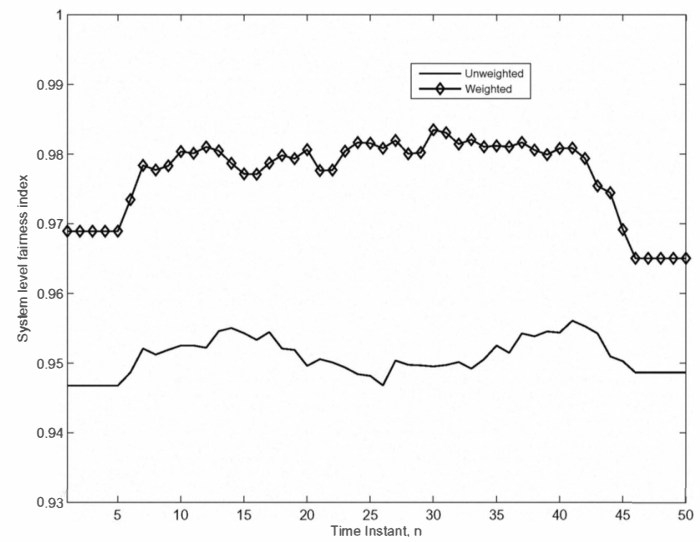

Fig. 5. Comparison of short term averaged system level fairness index (based on rate) of weighted and unweighted resource allocation schemes.

[6] Y. Xing, R. Chandramouli, S. Mangold and S. S. Nandagopalan, "Dynamic Spectrum Access in Open Spectrum in Wireless Networks," IEEE Journal on Selected Areas in Communications, Vol. 24, No. 3, pp. 627-637, March, 2006.

[7] Z. Ma, Z. Cao and W. Chen, "A Fair Opportunistic Spectrum Access (FOSA) Scheme in Distributed Cognitive," IEEE International Conference on Communications, May, 2008.

[8] C. Peng, H. Zheng and B. Y. Zhao, "Utilization and Fairness in Spectrum Assignment for Opportunistic Spectrum Access," Mobile Networks and Applications, May, 2006.

[9] J. Tang, S. Misra and G. Xue, "Joint Spectrum Allocation and Scheduling for Fair Spectrum Sharing in Cognitive Radio Wireless Networks," Computer Networks, April, 2008.

[10] D. Julian, M. Chiang, D. O'Neill and S. Boyd, "QoS and Fairness Constrained Convex Optimization of Resource Allocation for Wireless Cellular and Ad Hoc Networks," IEEE INFOCOM, June, 2002.

[11] S. Boyd and L. Vandenberghe, "Convex Optimization," Cambridge University Press, Cambridge, UK, 2004.

[12] T. Marler, "A Study of Multiobjective Optimization Methods for Engineering Application," PhD Thesis, The University of Iowa, May, 2005 , USA.

[13] Y. Xing and R. Chandramouli, "Human Behavior Inspired Cognitive Radio Network Design," IEEE Communications Magazine, December, 2008.

[14] H. Gintis, "Game Theory Evolving: A Problem-Centered Introduction to Modeling Strategic Behaviour," published by Princeton Univ. Press, 2000. 